\title{
Neonatal Ureaplasma parvum meningitis: a case report and literature review
}

\author{
Qiu Wang ${ }^{1 \#}$, Kai Wang ${ }^{1 \#}$, Yuanbo Zhang ${ }^{1}$, Chaosheng Lu' ${ }^{1}$, Yana Yan ${ }^{1}$, Xiaoxia Huang ${ }^{1}$, Jing Zhou ${ }^{2}$, \\ Lijiang Chen ${ }^{3}$, Dan Wang' \\ ${ }^{1}$ Department of Pediatrics, ${ }^{2}$ Department of Obstetrics, ${ }^{3}$ Clinical Laboratory, The First Affiliated Hospital of Wenzhou Medical University, Wenzhou \\ 325000, China \\ \#These authors contributed equally to this work. \\ Correspondence to: Dan Wang, MD. Department of Pediatrics, The First Affiliated Hospital of Wenzhou Medical University, No. 2 Fuxue Road, \\ Wenzhou 325000, China. Email: wd2014@126.com.
}

\begin{abstract}
Ureaplasma parvum (U. parvum) is common commensal in the female genitourinary tract. Despite $U$. parvum has been associated with chorioamnionitis, abortion, prematurity and perinatal complications, the invasive central nervous system (CNS) infection is rare in neonates. Diagnosis of $U$. parvum meningitis can be difficult for the atypical presentations and sterile cultures by conventional methods. Metagenomic nextgeneration sequencing (mNGS) could identify a broad range of human pathogens in a target-independent manner. Here, we performed mNGS to search for the infectious etiology in a term infant presenting with fever and seizure. $U$. parvum genome was identified by mNGS and further confirmed by PCR in the same cerebrospinal fluid (CSF) sample. As the quick and timely diagnosis, the baby was successfully treated with erythromycin for 4 weeks without complication. The clinical follow-up has showed that the physical and mental development are normal. In conclusion, mNGS may a promising diagnostic technology for $U$. parvum meningitis. As mNGS is able to identify diverse microbes in a single run, it could be a useful strategy to detection the clinical causative pathogens with atypical features in neonates.
\end{abstract}

Keywords: Neonate; meningitis; metagenomic next-generation sequencing (mNGS); Ureaplasma parvum (U. parvum); case report

Submitted Nov 29, 2019. Accepted for publication Jan 18, 2020.

doi: $10.21037 /$ tp.2020.02.04

View this article at: http://dx.doi.org/10.21037/tp.2020.02.04

\section{Introduction}

Ureaplasma species (spp.), including Ureaplasma urealyticum (U. urealyticum) and Ureaplasma paroum (U. parvum), are the smallest free-living organisms, lacking cell-wall, colonizers of the mucosal epithelial cells of genitourinary tract (1). They have been associated with a serial of obstetrical complications, including chorioamnionitis, premature rupture of membranes, prematurity, miscarriage, still birth, and perinatal morbidity and mortality $(1,2)$. Since the first case report of $U$. urealyticum meningitis in newborn in 1986, several researches have been indicated that $U$. urealyticum could be a causative pathogen of invasive disorders, such as pneumonia, bacteremia and central nervous system (CNS) infection $(2,3)$. However, the invasive infection in neonates with $U$. parvum has been rarely presented. The diagnosis of $U$. parvum meningitis is complicated by the atypical manifestations and the inherent biologic properties of this organism. U. parvum cannot be demonstrated on Gram staining and may not grow in conventional culture media. Unlike traditional assessment for specific pathogen, metagenomic next-generation sequencing (mNGS) screens for nearly all potential CNS infections and can identify novel or unexpected microbes. mNGS is being used increasingly for the clinical diagnosis of CNS infection in adults. Here, we present a rare case of $U$. parvum meningitis 




Figure 1 Sequence reads mapped to $U$. paroum by mNGS data. A total of 188 reads mapped to $U$. parvum in the reference database, corresponding to a total coverage of $1.24 \%$.

diagnosed by mNGS in a neonate. As the timely diagnosis, the baby was successfully treated with erythromycin without complication. This report aims to discuss the characteristics of neonatal $U$. parvum meningitis, including clinical features, new methods for microbial diagnosis, pathogenicity, and the challenges of appropriate treatment. We present the following case in accordance with the CARE Guideline (4).

\section{Case presentation}

A 11-day-old male infant (birth weight 3,390 g) was admitted to hospital for a sudden onset of fever $\left(38.7^{\circ} \mathrm{C}\right)$ and floppy. The baby was born by spontaneous vaginal delivery to a 41-year-old gravida 0 para 0 mother at 40 weeks of gestation. He did not have surface anomalies and was vigorous at birth with Apgar scores of 8 at $1 \mathrm{~min}$ and 9 at $5 \mathrm{~min}$. Maternal pregnancy was complicated by gestational diabetes mellitus and premature rupture of the membranes for 40 hours. The Blood analysis revealed leukocytosis $\left(15,740 / \mathrm{mm}^{3}\right)$, and elevated C-reactive protein $(10.4 \mathrm{mg} / \mathrm{L})$ with normal level of procalcitonin $(0.04 \mathrm{ng} / \mathrm{mL})$. Cerebrospinal fluid (CSF) assessment showed leukocytosis $\left(410 \mathrm{WBC} / \mathrm{mm}^{3}\right.$ with $55 \%$ of neutrophils), elevated protein level $(2.0 \mathrm{~g} / \mathrm{L})$, and reduced glucose $(<1.11 \mathrm{mmol} / \mathrm{L})$. Combined treatments of intravenous oxacillin $(50 \mathrm{mg} / \mathrm{kg} / \mathrm{dose}, \mathrm{q} 8 \mathrm{~h})$ and sulperazon $(50 \mathrm{mg} / \mathrm{kg} /$ dose, $\mathrm{q} 8 \mathrm{~h}$ ) were started for bacterial meningitis. No microorganism was detected on Gram staining or cultures of blood and CSF samples. On the day 4 of hospitalization, the infant presented local seizures and his anterior frontal became full and bulging. A cranial magnetic resonance image (MRI) identified ventriculitis and mild dilatation of right lateral ventricle, but no abscess or bleeding. A new CSF was analyzed and results showed $880 \mathrm{WBC} / \mathrm{mm}^{3}$ (48\% of neutrophils), $2.6 \mathrm{~g} / \mathrm{L}$ protein, and $<1.11 \mathrm{mmol} / \mathrm{L}$ glucose. Antibiotic therapy was modified to intravenously administered vancomycin $(15 \mathrm{mg} / \mathrm{kg} /$ dose, $\mathrm{q} 8 \mathrm{~h})$ and meropenem $(30 \mathrm{mg} / \mathrm{kg} / \mathrm{dose}, \mathrm{q} 8 \mathrm{~h})$; and phenobarbital was added to suppress seizure. Again, bacteriological cultures of blood and CSF still negative. After 7 days treatment of vancomycin and meropenem, the infant had a sustained fever of $38.0^{\circ} \mathrm{C}$ and somnolent, a third CSF was collected, which showed $205 \mathrm{WBC} / \mathrm{mm}^{3}$ ( $44 \%$ of neutrophils), protein at $1.9 \mathrm{mg} / \mathrm{L}$, and glucose at 1.9 $\mathrm{mmol} / \mathrm{L}$. Given CSF culture failed to reveal a pathogen, a CSF sample was collected and sent for the unbiased mNGS (BGI, Shenzhen, China), with a classification reference databases downloaded from NCBI (ftp://ftp.ncbi.nlm.ni-h. gv/genomes/) containing 1,424 whole genome sequence of viral taxa, 2,406 bacterial genomes or scaffolds, 199 fungi related to human infection and 135 parasites associated with human diseases (5). Within 72 hours, the mNGS produced 36.1 million reads and detected 188 reads of $U$. parvum, covering $1.24 \%$ $(9,306 / 751,719)$ of the $U$. parvum genome (Figure 1). To further verify the presence of this pathogen, a specific PCR analysis was conducted with the same CSF sample and the PCR product was assessed by Sanger sequencing. The PCR primers were 5'-CATTGATGTTGCACAAGGAGAAA-3' and 5'-TTA GCACCAACATAAGGAGCTAAATC-3', which target a $U$. parvum specific region of $16 \mathrm{~S}$ rRNA gene. Based on these results, the patient was diagnosed of $U$. parvum meningitis and was administered with intravenous erythromycin $(10 \mathrm{mg} / \mathrm{kg} / \mathrm{dose}, \mathrm{q} 6 \mathrm{~h})$; other antibiotics were weaned. On day 3 and 7 of erythromycin treatment, the fever subsided and CSF test recovered, respectively; and his condition continued favorably. After the 4 weeks course of erythromycin, the repeated CSF was without biochemical abnormalities and baby was discharged home. The timeline of this case was shown in Figure 2. The infant developed well without abnormal neurological signs during the follow-up until 18 months old. His head MRI at the age of 6 months showed the resolution of ventriculitis and hydrocephalus. 


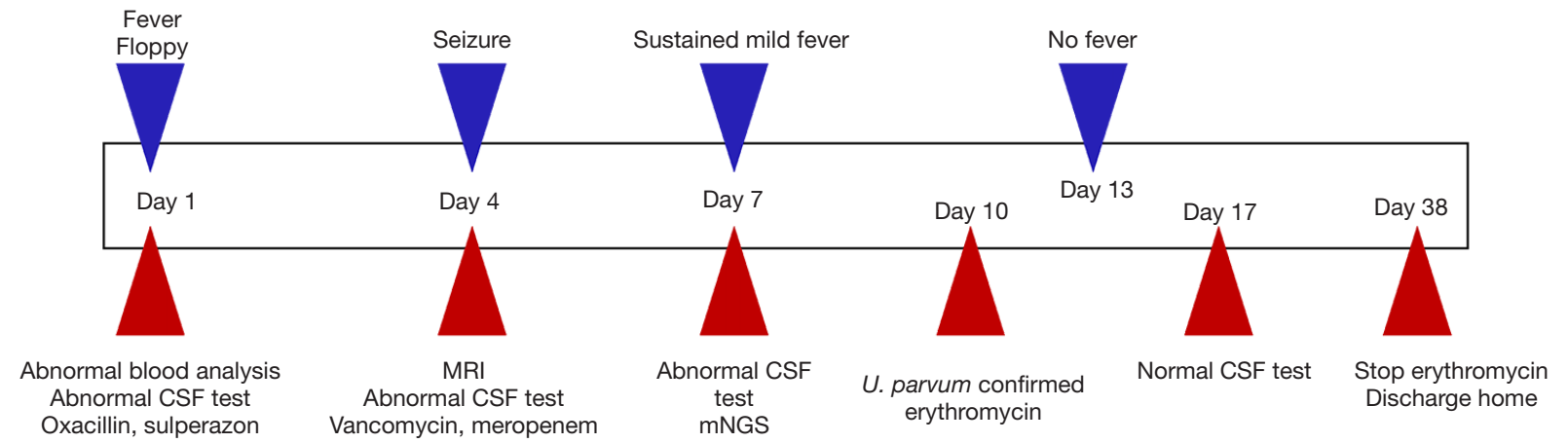

Figure 2 The timeline of this case, including clinical presentations, tests and treatments.

Table 1 Characteristics of published cases with Ureaplasma parvum meningitis

\begin{tabular}{|c|c|c|c|c|c|c|c|c|}
\hline No. & Gender & Onset time & Presentation & Identification & Treatment & Complication & Others & Ref \\
\hline 2 & Female & $\begin{array}{l}4 \text { weeks after } \\
\text { birth }\end{array}$ & $\begin{array}{l}\text { HCP and developmental } \\
\text { delay }\end{array}$ & PCR & $\mathrm{CHL} \times 3$ weeks & $\begin{array}{c}\text { HCP and } \\
\text { developmental delay }\end{array}$ & $\begin{array}{l}\text { Preterm, maternal } \\
\text { cervical insufficiency } \\
\text { and cerclage }\end{array}$ & (6) \\
\hline 4 & Male & $\begin{array}{c}16 \text { days after } \\
\text { surgery }\end{array}$ & $\begin{array}{l}\text { Fever, headache, } \\
\text { photophobia and } \\
\text { meningeal irritation }\end{array}$ & PCR & LVFX $\times 3$ weeks & None & $\begin{array}{c}\text { Adult, } \\
\text { craniopharyngioma } \\
\text { ablation }\end{array}$ & (8) \\
\hline
\end{tabular}

IVH, intraventricular hemorrhage; HCP, hydrocephalus; ERY, erythromycin; CIP, ciprofloxacin; CHL, chloramphenicol; THI, thiophenicol; LVFX, levoflfloxacin.

\section{Discussion}

Here, we describe a rare case of neonatal meningitis resulting from $U$. parvum infection. Like the most previous cases of $U$. parvum meningitis (6-9), the common cultures of blood and CSF were negative. But after the pathogen was identified by mNGS and confirmed by PCR, the baby was successfully treated with erythromycin.

Despite $U$. parvum has been consistently associated with chorioamnionitis, abortion, stillbirth, premature rupture of membranes and prematurity.

Despite Ureaplasma spp have been consistently associated with histological chorioamnionitis, abortion, stillbirth, preterm birth and perinatal complications, Despite Ureaplasma spp have been consistently associated with histological chorioamnionitis, abortion, stillbirth, preterm birth and perinatal complications.

Despite $U$. parvum has been consistently associated with histological chorioamnionitis, abortion, stillbirth, preterm birth and perinatal complications $(1,2)$, the invasive infection is rare. According to the literature, there are four cases of $U$. parvum meningitis have been previously reported (Table 1) (6-9). One is in an adult after surgical ablation of a craniopharyngioma (9). Other three cases are in neonates (two prematurity and one term) (6-8). In all reported neonatal cases, no other pathogen was found despite extensive microbiological diagnostics. Infection with $U$. parvum in neonates has been documented to be late onset meningitis and has variable clinical presentation including fever, apnea, cardiovascular instability, rhinitis, conjunctivitis, seizure and hydrocephalus. In this case, the full-term boy also had manifestations of fever, floppy and seizure. The results of initial CSF analysis might with the predominance of leukocytes or lymphocytes and the glucose 
level could be undetectable or normal, suggesting bacterial or viral meningitis. Similarly, the CSF was presented with pleocytosis and decreased glucose level in our case. Thus, the CSF profile of $U$. parvum meningitis may be various and atypical. After the specific treatment of $U$. parvum, the CSF profile has been reported to gradually normalize, paralleled by a continuous clinical improvement. Development of hydrocephalus with or without of hemorrhage also has been reported in all neonatal cases, either at initial signs or in the clinical course. And the long-term outcome of neonatal $U$. parvum meningitis ranges from complete resolution to progressive internal hydrocephalus or neurodevelopmental impairment $(6,8)$. In accordance to the previous $U$. parvum meningitis, the hydrocephalus gradually resolved and the baby boy develops appropriately in this case. Only one previous case which manifested with sustained meningitis and progressive hydrocephalus had poor neurological outcome $(6,8)$, implying that continuous inflammation is related to the adverse outcome. Thus, early diagnosis and initiation of appropriate treatment are necessary to improving the prognosis.

Although Ureaplasma normally exist in a state of adherence to mucosal epithelial cells, they can disseminate to other sites and cause invasive infection when there is a disruption of the mucosa and/or an underlying defect in host defenses, such as in the developing fetus or newborn. Their pathogenicity has been imputed to inflammatory response by modulating the immune system in a complex way, not only promoting pro-inflammation, but also inhibit immune response $(1,10)$. The balance between pro-inflammatory cytokines (e.g., tumor necrosis factor- $\alpha$, interleukin (IL)$1 \beta$, IL-2, IL-6, IL-8) and anti-inflammatory cytokines (e.g., IL-10) is disturbed by Ureaplasma infection (1). In addition, they could also affect innate host immune by downregulating cell defense genes (e.g., antimicrobial peptides), decomposing the host cell membrane via phospholipase, and exerting cytotoxicity through ammonia accumulation or PH-shift via hydrolyzing urea $(11,12)$.

In the previous $U$. parvum meningitis cases, the pathogen in CSF was demonstrated by PCR in three cases and by the combination with PCR and culture in on case. Ureaplasma are the smallest free-living organisms. They cannot be visualized by Gram stain since the lacking of cell wall (13). Given they are not surviving in standard culture media, in order to harvest these organisms, clinical specimens should be inoculated immediately onto specialized culture media before they are allowed to dry $(7,13)$. And culture detection take long time intervals and positive rate can be as low as
$40 \%$ (14). On account of the complex growth requirements and specific culture condition, the organism might avoid detection. Broad range bacterial PCR is another method for the Ureaplasma identification and superior to traditional culture methods. But this hypothesis-driven molecular testing may miss the pathogen when there is no consideration about the specifically targeted organisms or when the used primers contain mismatches to the microbial strain involved, which decreases the sensitivity of detection. Above options for diagnosing infections caused by Ureaplasma are limited due to the shortcomings of both culture and PCR-based techniques. Thus, microbial diagnostics on Ureaplasma may be delayed by the difficulties in pathological detection. Here, we found $U$. parvum in CSF by mNGS analysis. mNGS, a high-throughput or massively parallel sequencing method, is beneficial for diagnosis in patients with suspected infectious but negative results for conventional assessments (5). It could apply for hypothesis-free and universal pathogen detection, promising to improve infectious disease diagnostics, especially in immunocompromised neonates or critically ill patients. This novel technique will enable timely diagnosis and treatment of invasive $U$. parvum infection and is expected to result in excellent outcomes, as presented in this case. However, mNGS method also has inherent disadvantages, including sequencing human host gene background, suffering from the contamination with environmental species, and the expensive cost.

The treatment of invasive Ureaplasma infection in neonates is particularly challenging. Previous cases of $U$. parvum meningitis shows several therapeutic chemicals (Table 1). Three reports in neonates used different antibiotic regimes: two preterm infants were treated by chloramphenicol and the combination of erythromycin and ciprofloxacin, respectively; the other term baby was administered with ciprofloxacin and thiophenicol. Considering that Ureaplasma are free of cell wall, the antibiotics targeting protein or DNA synthesis may effective, such as macrolides, tetracyclines, clindamycin, chloramphenicol and quinolones (14). However, the potential side effects of above drugs have been taken into consideration when used in the newborn. Tetracyclines can induce permanent dental discolorations or skeletal abnormalities; chloramphenicol may cause "gray baby syndrome" or blood dyscrasias; and fluoroquinolones have arthropathogenic effects. Thus, these drugs are generally avoided in neonates due to concerned toxicities. Macrolide antibiotics are usually used in the Ureaplasma treatment. Here, we firstly treated the baby with erythromycin. Erythromycin is not suggested for the treatment of 
Ureaplasma meningitis due to its poor liquor penetrance (14). But some cases have been successfully treated with erythromycin (15), which may be explained by the increase of blood-brain barrier permeability when meningitis is occurring. In addition, the appropriate duration of antibiotic therapy for Ureaplasma meningitis is not well understood. We choose erythromycin for 4 weeks, as is general practice in the treatment of other types of bacterial meningitis in neonates. In other reports, the treatment duration may range from 2 to 6 weeks in infants (16).

In conclusion, neonatal Ureaplasma meningitis might be more prevalent than previously suspected. Its presentations and CSF changes may be not typical. Any infant suffering from internal hydrocephalus, with evidence of congenital infection, or with CSF pleocytosis, should be recommend to have CSF microbial diagnostics on Ureaplasma. Early diagnosis and initiation of appropriate treatment could prevent the adverse outcome. Identification of $U$. parvum can be difficult for it may escape common detection due to complex culture media and condition. mNGS can identify diverse microbes in one run, it could be a promising strategy to diagnosis $U$. parvum meningitis or detect the causative pathogens with atypical clinical features in neonates.

\section{Acknowledgments}

Funding: This work was supported by the National Natural Science Foundations of China (81701485) and the Science and Technology Municipal Commission of Wenzhou (Y20170007, Y20160021).

\section{Footnote}

Conflicts of Interest: All authors have completed the ICMJE uniform disclosure form (available at http://dx.doi. org/10.21037/tp.2020.02.04). KW serves as the unpaid section editor of Translational Pediatrics from Oct 2019 to Dec 2020. The other authors have no conflicts of interest to declare.

Ethical Statement: The authors are accountable for all aspects of the work in ensuring that questions related to the accuracy or integrity of any part of the work are appropriately investigated and resolved. This study was approved by the ethical review committee of The First Hospital Affiliated to Wenzhou Medical University. Written informed consent was obtained from the patient for publication of this Case report and any accompanying images.
Open Access Statement: This is an Open Access article distributed in accordance with the Creative Commons Attribution-NonCommercial-NoDerivs 4.0 International License (CC BY-NC-ND 4.0), which permits the noncommercial replication and distribution of the article with the strict proviso that no changes or edits are made and the original work is properly cited (including links to both the formal publication through the relevant DOI and the license). See: https://creativecommons.org/licenses/by-nc-nd/4.0/.

\section{References}

1. Silwedel C, Speer CP, Glaser K. Ureaplasma-associated prenatal, perinatal, and neonatal morbidities. Expert Rev Clin Immunol 2017;13:1073-87.

2. Oh KJ, Romero R, Park JY, et al. The earlier the gestational age, the greater the intensity of the intraamniotic inflammatory response in women with preterm premature rupture of membranes and amniotic fluid infection by Ureaplasma species. J Perinat Med 2019;47:516-27.

3. Gwee A, Chinnappan M, Starr M, et al. Ureaplasma meningitis and subdural collections in a neonate. Pediatr Infect Dis J 2013;32:1043-4.

4. Riley DS, Barber MS, Kienle GS, et al. CARE guidelines for case reports: explanation and elaboration document. J Clin Epidemiol 2017;89:218-35.

5. Gu W, Miller S, Chiu CY. Clinical metagenomic nextgeneration sequencing for pathogen detection. Annu Rev Pathol 2019;14:319-38.

6. Glaser K, Wohlleben M, Speer CP. An 8-month history of meningitis in an extremely low birth weight infant? Longlasting infection with Ureaplasma parvum. Z Geburtshilfe Neonatol 2015;219:52-6.

7. Clifford V, Tebruegge $\mathrm{M}$, Everest $\mathrm{N}$, et al. Ureaplasma: pathogen or passenger in neonatal meningitis? Pediatr Infect Dis J 2010;29:60-4.

8. Biran V, Dumitrescu AM, Doit C, et al. Ureaplasma paroum meningitis in a full-term newborn. Pediatr Infect Dis J 2010;29:1154.

9. Pailhoriès $\mathrm{H}$, Chenouard R, Eveillard M, et al. A case of Ureaplasma parvum meningitis in an adult after transphenoidal ablation of craniopharyngioma. Int J Infect Dis 2019;84:5-7.

10. Glaser K, Silwedel C, Waaga-Gasser AM, et al. Ureaplasma isolates differentially modulate growth factors and cell adhesion molecules in human neonatal and adult monocytes. Cytokine 2018;105:45-8. 
11. Wang X, Greenwood-Quaintance KE, Karau MJ, et al. Ureaplasma parvum causes hyperammonemia in a pharmacologically immunocompromised murine model. Eur J Clin Microbiol Infect Dis 2017;36:517-22.

12. Xiao L, Crabb DM, Dai Y, et al. Suppression of antimicrobial peptide expression by Ureaplasma species. Infect Immun 2014;82:1657-65.

13. Waites KB, Katz B, Schelonka RL. Mycoplasmas and Ureaplasma as neonatal pathogens. Clin Microbiol Rev 2005;18:757-89.

Cite this article as: Wang Q, Wang K, Zhang Y, Lu C, Yan Y, Huang X, Zhou J, Chen L, Wang D. Neonatal Ureaplasma parvum meningitis: a case report and literature review. Transl Pediatr 2020;9(2):174-179. doi: 10.21037/tp.2020.02.04
14. Glaser K, Speer CP. Neonatal CNS infection and inflammation caused by Ureaplasma species: rare or relevant? Expert Rev Anti Infect Ther 2015;13:233-48.

15. Waites KB, Rudd PT, Crouse DT, et al. Chronic Ureaplasma urealyticum and Mycoplasma bominis infections of central nervous system in preterm infants. Lancet 1988;1:17-21.

16. Ku LC, Boggess KA, Cohen-Wolkowiez M. Bacterial meningitis in infants. Clin Perinatol 2015;42:29-45. 